\title{
Management of paediatric diabetic ketoacidosis: are we doing it right?
}

Manisha Ramphul and Babita Khetriwal

Lister Hospital, East and North Hertfordshire NHS Trust, Stevenage, England, SG1 4AB

\section{Introduction}

Diabetic ketoacidosis (DKA) is a medical emergency, requiring careful fluid management and insulin administration. The guidelines published by the British Society for Paediatric Endocrinology and Diabetes (BSPED) in 2009 aim to standardise the approach to the management of paediatric DKA across NHS hospitals. Our aim was to report our experience in the management of DKA in children in a district general hospital setting.

\section{$\underline{\text { Method }}$}

Retrospective case review of 19 cases of paediatric DKA (patients aged $<19$ years) presenting to a district general hospital between January 2012 and December 2012.

\section{$\underline{\text { Results }}$}

The median age of presentation was 15 years (interquartile range 11.5 to 16.5 years). Figure 1 shows the proportion of children in this study by age.
The history and general examination findings were appropriately documented for all patients assessed. Blood glucose, ketones and $\mathrm{pH}$ were measured in all children. The weight was only documented in 12 patients (63\%). The hydration status was documented in 7 children (36\%) only. The timing of insulin was appropriate in 16 patients (84\%). 2 children (10\%) were inappropriately given fluid boluses.

Once treatment was initiated, blood tests were monitored as per the BPSED protocol in 13 patients $(68 \%)$. The average length of stay in hospital was 3.5 days. All patients and their families received a discharge planning meeting and further education.

Prior to the introduction of the Integrated Care Pathway for paediatric DKA (DKA ICP) in our Trust, doctors were trained in how to use it in their practice. Despite this, the DKA ICP was only used in 3 patients in our study group (16\%).

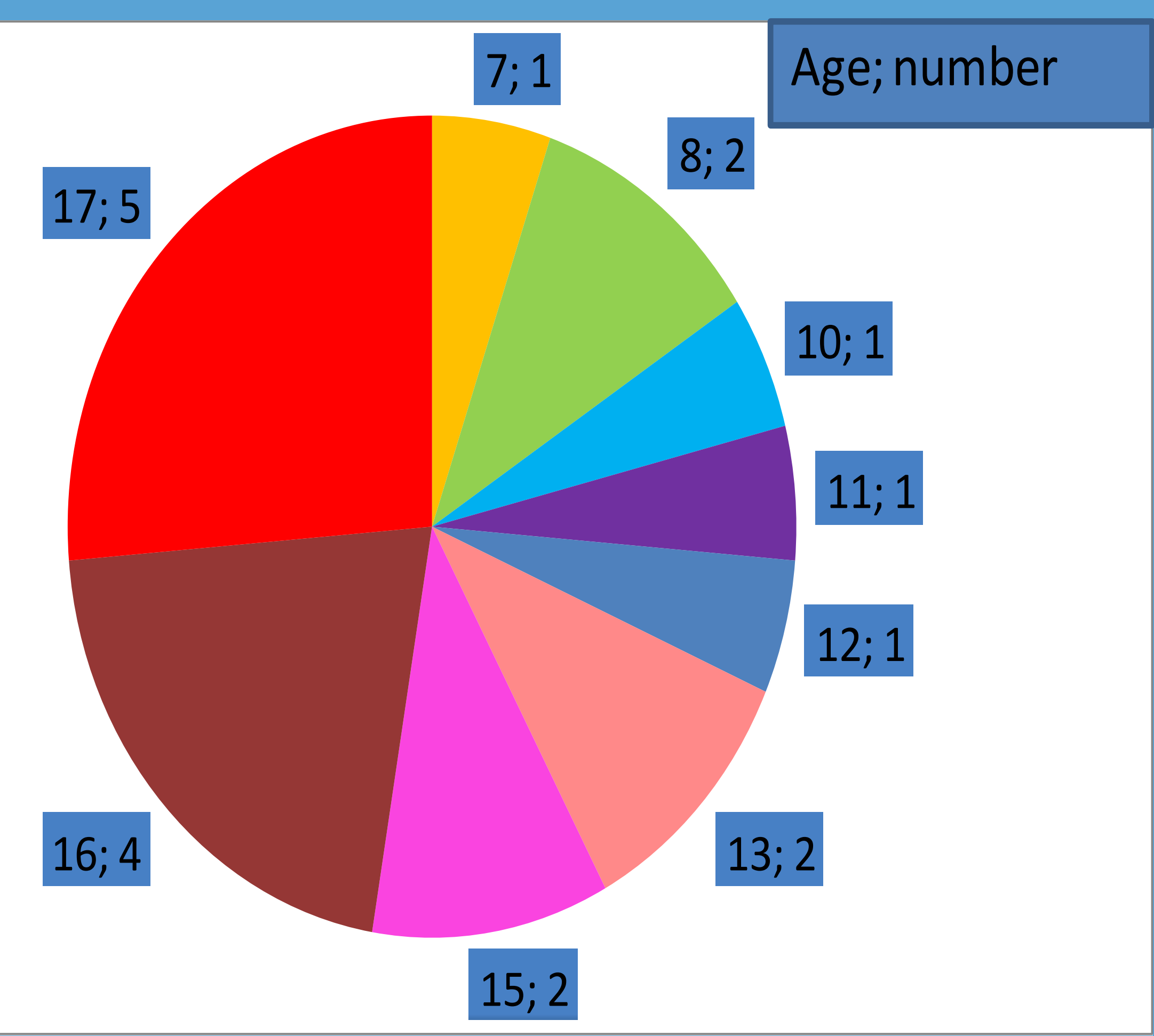

Figure 2 shows our performance in the different areas of the assessment and management of DKA.

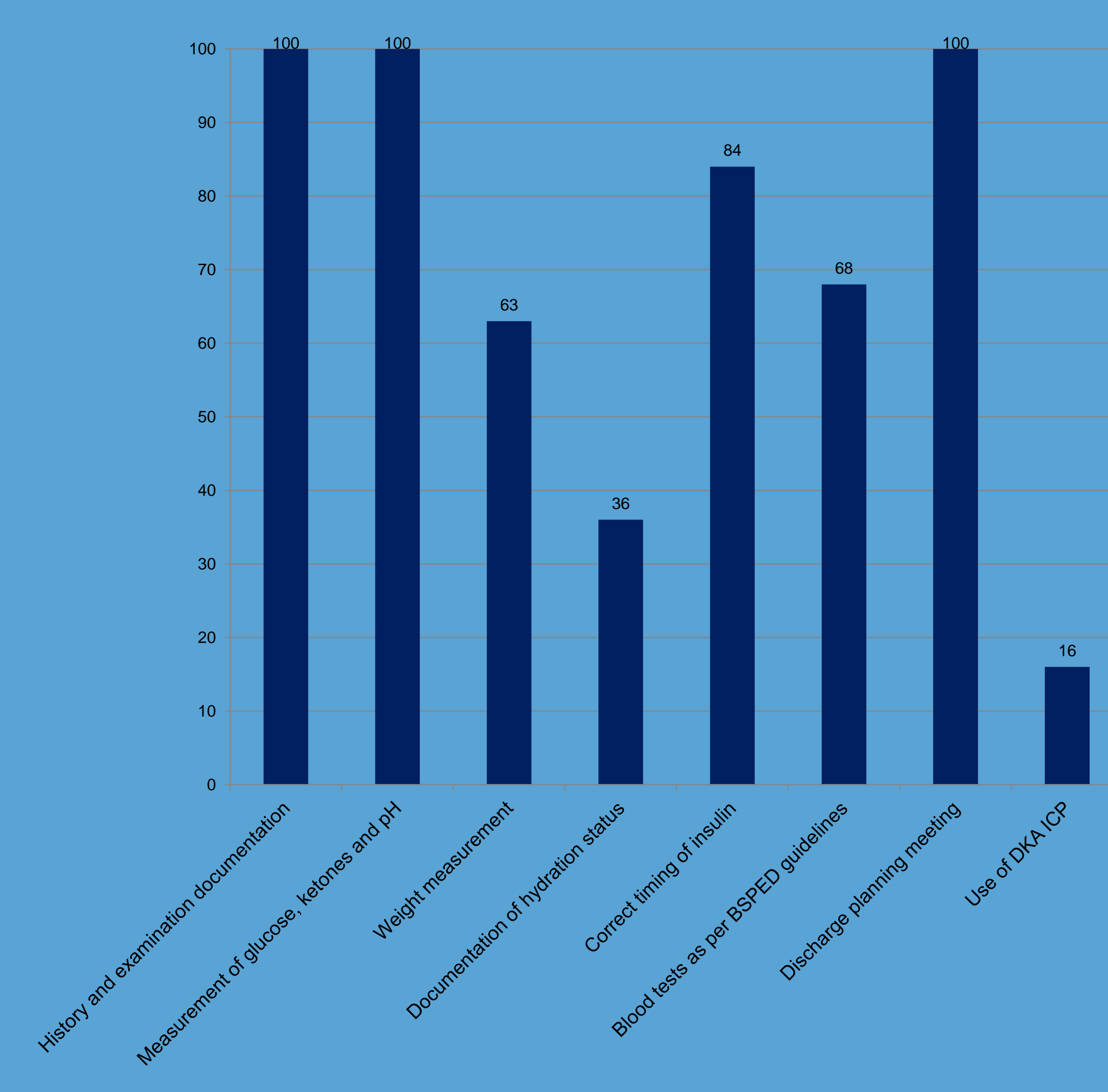

Figure 2: Chart showing the percentage of children in whom different elements, of DKA assessment and management, were achieved

\section{Conclusion}

The management of DKA is still suboptimal despite robust guidelines which have been put in place. Children with diabetes commonly present to their local district general hospitals in emergencies such as DKA.

Hence we believe that paediatric trainees nationwide should receive further interactive education on the management of DKA. The use of the DKA ICP, devised to reduce errors in fluid calculations, should be encouraged across all NHS trusts. 